\title{
ENHANCEMENT OF X-RAY LINE ENERGY SPECTRA FROM A GAS MICROSTRIP DETECTOR
}

\author{
J.E.Bateman \\ Rutherford Appleton Laboratory, Chilton, Didcot, Oxon, OX11 0QX, U.K.
}

12 June 2000

\begin{abstract}
It is shown that the stable line response function produced by a gas microstrip detector operated in "wall-less" mode permits mathematical manipulation, which greatly facilitates analysis of the data and explicit enhancement of x-ray line spectra. As a result, given adequate counting statistics, effective energy resolution comparable to that of a cryogenic silicon or germanium detector may be achieved.
\end{abstract}




\section{Introduction}

In energy-dispersive $\mathrm{x}$-ray spectroscopy the gas proportional counter has been largely superseded by the cryogenic semiconductor ( $\mathrm{Ge}$ or $\mathrm{Si}$ ) detector which can give a FWHM energy resolution down to the order of $150 \mathrm{eV}$; some five or so times better than that of the gas counter in the few-keV energy range. The main disadvantages of this device are the complexity and cost of the cryostat necessary for operation. Technical limitations arise from the finite front contact thickness (which raises the useful threshold of operation to $\approx 1 \mathrm{keV}$ ) and the slow amplifier time constants necessary to achieve the low noise (which limit the data capture rates to $\approx 100 \mathrm{kHz}$ ). At low x-ray energies the gas counter has certain operational advantages which can be used to advantage, such as flexibility of gas filling, the ability to function with a very thin window (or no window with internal counting) and the ability to detect x-rays below $1 \mathrm{keV}$. Compared to the conventional wire proportional counter, the advent of the Gas Microstrip Detector (GMSD) [1] has the added extra advantages of very high counting rates and a flexible geometry.

It has been shown elsewhere [2] that it is possible to adapt the geometry of the GMSD to gain freedom from the spectrum-distorting effect of partially collected events occurring near the detector edges and so achieve "wall-less" operation. The important consequence of this is that very clean pulse height spectra can be obtained which fit well to a standard line response function (LRF) in the form of a lognormal (LN) curve. Fitting this LRF to elemental emission lines which would not normally be resolved by the GMSD identifies the line positions with errors of a few $\mathrm{eV}$. This is achieved at a cost of excess noise in the amplitude of the fitted lines which requires additional counting statistics to correct [2]. Since the GMSD is an intrinsically high rate device, using this technique, it can in many situations, deliver the higher statistics necessary to achieve the improved energy resolution.

In the many situations in which the x-ray signal is confined to fixed lines (e.g. x-ray fluorescence analysis) the fitting procedure is quite adequate. However, in situations where the x-ray spectrum does not consist chiefly of lines, or if the lines are unknown, fitting is not possible. The question now arises as to whether the stable LRF of the GMSD can be used more generally in an enhancement algorithm which can process the measured pulse height spectrum and deliver the equivalent spectrum of a pseudodetector with improved energy resolution. In this report it is shown that such processing is indeed feasible and offers the possibility of useful application.

\section{The Gas Microstrip Detector}

The GMSD consists of an array of fine metallic lines produced on a semiconducting glass substrate by standard micro-lithographic processes. Connected alternately to a high electrical potential (anodes and cathodes) the metal strips function as amplifiers of free electrons in the gas surrounding them. A drift cathode at a suitable distance from the plate (typically $10 \mathrm{~mm}$ ) defines the gas volume from which $\mathrm{x}$-ray-induced electron clouds are collected onto the anodes for amplification. Gas gains of up to 5000 enable individual $\mathrm{x}$-rays to be detected as a pulse in an amplifier connected to either a single strip or groups of strips. The cylindrical geometry of the conventional proportional counter is replaced by planar geometry which is yet further simplified by the weak dependence of the gas gain on the drift electrode position [3]. A 
considerable advantage of the GMSD is its high rate capability. Using the divided electrode structure it is possible to achieve counting rate densities of $\approx 10 \mathrm{MHz} / \mathrm{cm}^{2}$, giving several tens of $\mathrm{MHz}$ for a typical detector area of several $\mathrm{cm}^{2}$ [4].

By making the x-rays enter the drift space parallel to the electrode planes, avoiding the regions very close to the electrode planes $(\approx 1 \mathrm{~mm})$ and using anti-coincidence guard sections on either side of the active region of the detector, "wall-less" operation can be achieved. Figure 1 shows the pulse height spectrum obtained from this set-up when the x-rays from an ${ }^{55} \mathrm{Fe}$ internal conversion source are detected in a GMSD filled with an argon-based gas mixture. Reference [2] gives more details of the set-up.

\section{X-ray Line Detection in the "Wall-less" GMSD}

Figure 1 shows the $\mathrm{LN}$ fits to the $\mathrm{K}_{\alpha}$ and $\mathrm{K}_{\beta} \mathrm{Mn} \mathrm{x}$-ray lines (5.9keVand $6.49 \mathrm{keV}$ ) and the argon escape lines $(2.9 \mathrm{keV}$ and $3.49 \mathrm{keV})$ as observed in a GMSD with $10 \mu \mathrm{m}$ wide anodes and $90 \mu \mathrm{m}$ wide cathodes with a $100 \mu \mathrm{m}$ separation. The filling gas is argon $+25 \%$ isobutane and the drift electrode is spaced at $10 \mathrm{~mm}$. The counter is operated in wall-less mode with the discriminator levels set at $0.59 \mathrm{keV}$ in the anticoincidence guard channels. The fitting is extremely close with the fitted peak channels accurately linear with the known x-ray line energies [2]. The parametric form of the $\mathrm{LN}$ distribution used is:

$$
\frac{d n}{d x}=\frac{a}{\sqrt{2 \pi} b x} \exp \left(\frac{(\ln (x)-\ln (c))^{2}}{2 b^{2}}\right)
$$

where $x$ is the pulse height analyser (PHA) channel number, $a$ is the total number of counts in the line, $b$ is the standard deviation (SD) of the $\ln (x)$ distribution (i.e. the relative SD of the $x$ distribution) and $\ln (c)$ is the mean of the $\ln (x)$ disribution. The errors dictated by the statistical noise are evaluated by the Marquart-Levenburg fitting routine and displayed in figure 1. (The Marquardt-Levenberg algorithm is used in the implementation in the commercial EasyPlot package [5].)The position errors are 0.097channels $(2.2 \mathrm{eV})$ in the $\mathrm{K}_{\alpha}$ and $0.28 \mathrm{ch}(6.4 \mathrm{eV})$ in the $\mathrm{K}_{\beta}$. The amplitude errors are 2183 counts (in $3.63 \times 10^{5}$ )for the $K_{\alpha}$ and 1972 counts (in 99439) in $K_{\beta}$.

In the fitted parameters of figure 1 two points are immediately noticeable; the position errors are very low, and the amplitude errors are high compared with the Poisson errors expected from the amplitudes i.e. 602 for $K_{\alpha}$ and 100 for $K_{\beta}(\sqrt{ } N)$. The factor of 3.63 increase in the SD of the fitted $\mathrm{K}_{\alpha}$ means that $13.1\left(3.63^{2}\right)$ times the number of counts must be collected to achieve the same amplitude resolution as would be achieved by a given number of events in the peak as detected by an ideal detector. The corresponding factor in the $\mathrm{K}_{\beta}$ case is 39.1 . The ratio of the observed variance in the fitted number of counts to the Poisson value (i.e. $\sigma_{N}{ }^{2} / N$ ) is known as the excess noise factor $(F)$ and it represents the factor by which the counting time must be extended to achieve the same resolution in the counts as would be achieved by an ideal detector counting $N$ events. $F$ varies with the partition fraction between two adjacent lines and also with the ratio of the line resolution to the line spacing and 
increases from unity as the fraction of the counts in the peak under study and the separation of the lines decrease [2].

The relative SD of the lognormal line response function (LRF) is assumed constant over the energy span of the $\mathrm{K}_{\alpha}$ and $\mathrm{K}_{\beta}$ lines $(0.59 \mathrm{keV})$. One can allow the parameter $b$ to fit separately to the $\mathrm{K}_{\beta}$ line but no significant improvement is seen. For the $\mathrm{K}_{\alpha}$ line $b=0.0606$ giving a relative $F W H M\left(2.36^{*} b^{*} 100\right)$ of $14.3 \%$. At the escape peaks $b=0.091$ giving a $F W H M$ of $21.5 \%$ showing the typical increase of the relative LRF width as the x-ray energy decreases.

The LN fitting process makes it easy to quantify the behaviour of the energy resolution as a function of X-ray energy. Figure 2 shows the measured FWHM derived in this way from the Mn K lines plus the fluorescent $\mathrm{K}$ lines from $\mathrm{Rb}$, Mo and $\mathrm{Ag}$ in the same (argon-filled) GMSD. The relationship found is [6]:

$$
F W H M=\sqrt{\frac{1061.5}{E_{x}}+5.14^{2}} \%
$$

which shows the $E_{x}^{-1 / 2}$ term dictated by ionisation statistics with a constant term of $5.14 \%$ which is the due to the gain variation of the counter over the working area. $E_{x}$ is the $\mathrm{x}$-ray line energy in $\mathrm{keV}$.

\section{The Enhancement of GMSD X-ray Line Spectra}

The characterisation of the line response function (LRF) summarised above provides all the necessary information for the exploration of enhancement algorithms. However, such algorithms are computationally intensive and the energy-dependent LRF which is implied by relations (1) and (2) above implies a frequent recalculation of the LRF within the kernal of the algorithm leading to an excessive demand for computing power. Transforming the spectrum into the space $u=x^{1 / 2}$ simplifies the situation and makes the programming more efficient. For convenience this process is called the U-transform.

\subsection{The U-transform}

Figure 3 illustrate one of the major problems associated with the x-ray pulse height spectra obtained from a gas counter. In this figure a montecarlo program has simulated the LN pulse height distributions produced by a GMSD in response to x-ray lines at $0.75,1.5,3.0$ and $6.0 \mathrm{keV}$. There are $10^{4}$ events in each line. The energy resolution of the detector is determined by the relation $F W H M=39.3 E_{x}^{-1 / 2} \%$ which is typical of gas counters. This translates to $\sigma_{R}=0.166 E_{x}^{-1 / 2}$ ( $b$ in equation (1)) and $\sigma_{x}=$ $0.166 E_{x}{ }^{1 / 2} \mathrm{keV}$. The effect of the energy dependence of the resolution is to make $\mathrm{x}$-ray lines of the same intensity appear very different. Making the transformation to the independent variable $u=x^{1 / 2}$, it is noted that a step in $u$-space $d u=1 / 2 x^{-1 / 2} d x$. If $d x$ is the width of the X-ray pulse height spectrum we see that $\sigma_{u}=1 / 2 x^{-1 / 2} \sigma_{x}$. However, $\sigma_{x}=k x^{1 / 2}$ (where $k$ is a constant) so that $\sigma_{u}=k / 2$ and is independent of the X-ray energy. The normalisation of the line distributions is assured by making the transformation of the ordinates of the pulse height spectrum according to the relation $d N / d u=2 u d N / d x$. 
Making this transformation of the data in figure 3 produces the spectrum in $u$-space as shown in figure 4 . Here it is observed that the line distributions are now all of the same width and amplitude.

Since the resolution parameter $b$ in equation (1) is the relative resolution $\left(\sigma_{R}\right)$, and the absolute resolution $\left(\sigma_{x}\right)$ is constant, $b$ is not independent of $u$ and must still be recalculated at every $x$ value. This problem is solved by noting the behaviour of the LN function (equation (1)) under the $u$-transformation. In this case $c \rightarrow c^{1 / 2}$ and $b \rightarrow$ $b / 2$, i.e. the width of the LN curve is halved. Since the skewness of the LN function is also determined by $b$ the halving of $b$ also increases the symmetry of the distribution. If one examines the behaviour of the LN distribution, one finds that as $b \rightarrow 0$ the function approaches a simple gaussian. Since $b$ is generally small $(<0.1)$ this means that, perhaps except at very low energies where $b$ is $>0.1$, the peaks in figure 4 can be approximated well by a gaussian function of constant width. This is the ideal situation for the application of a spectrum-enhancing algorithm. In figure 4 the precision of this approximation can be assessed by fitting the four peaks to a sum of four gausian functions with the same SD. As can be seen from the figure the result is very satisfactory with the peak areas fitting to $10^{4}$ with errors of $<1 \%$. When the squares of the distribution means are plotted against the input energies a perfect straight line is obtained.

In practical detectors there is always a constant term in the functional representation of the energy resolution (equation (2)) which spoils the exact relation $\sigma_{x}=k x^{1 / 2}$ on which the success of the U-transform depends. In the GMSD from which equation (2) was evaluated this contribution was $5.14 \%$ in the $F W H M$ or 0.022 in $\sigma_{R}$. This is small enough to be negligible until $E_{x}>20 \mathrm{keV}$. This figure represents an upper limit for the gain control on a GMSD plate: values of $<0.01$ have been observed [7].

Figure 5 shows the data of figure 1 plotted in u-space with gaussian functions fitted. The amplitudes obtained for the $\mathrm{K}_{\alpha}$ and $\mathrm{K}_{\beta}$ lines agree very well (within $1 \%$ ) with those obtained in $\mathrm{x}$-space with the $\mathrm{LN}$ fit. When the squares of the means of the fitted distributions are plotted against the known x-ray line energies an excellent straight line is obtained in figure 6 .

Applying the U-transform to a wider range of x-ray energies yields similarly satisfactory results. Figure 7 shows the fitting of a constant gaussian width to the pulse height spectrum given by an argon-filled GMSD when exposed to a silver fluorescence source. The Ag K lines are fitted along with the $\mathrm{Ni}, \mathrm{Fe}$ and $\mathrm{Cr} \mathrm{K}$ lines generated by the stainless steel body of the ${ }^{241} \mathrm{Am}$ source holder. A single gaussian $\mathrm{SD}$ fits all the lines between $4 \mathrm{keV}$ and $22 \mathrm{keV}$. Figure 8 shows the fitted peak positions $\left(u_{\text {peak }}^{2}\right)$ plotted against the line energies. There is some confusion in the $\mathrm{Fe}$ region with overlapping $\mathrm{K}_{\beta}$ lines, but taken as a whole the agreement is very good.

A U-transformed pulse height spectrum in the low-keV region is shown in figure 9. Here the $\mathrm{K}_{\alpha}$ lines of $\mathrm{Si}(1.74 \mathrm{keV}), \mathrm{Ar}(2.96 \mathrm{keV})$ and $\mathrm{Mn}(5.89 \mathrm{keV})$ are generated from a glass plate irradiated by an 55Fe source and detected in a GMSD with a $\mathrm{He} / \mathrm{Ne} /$ isobutane filling. Within the limits set by the poor statistics and the presence of low levels of other lines, a reasonable fit with a uniform, gaussian LRF is obtained. 


\subsection{Spectrum Enhancement}

Having reduced the enhancement problem to the deconvolution of a constant gaussian LRF the task becomes much easier. There is a considerable choice of algorithms which can be applied to this type of problem, for example, maximum entropy, maximum likelihood or simulated annealing. The simulated annealing (SA) algorithm used in this case is not particularly to be preferred and is used because the software was available.

The SA algorithm used is derived from the ideas developed in reference [8]. A target distribution (the final solution) is generated by adding or subtracting a small "grain" randomly across the field. The grain is chosen to be a small fraction of the maximum amplitude in the input distribution (the raw data), typically $1 \%$. After each addition the target distribution is convolved with the known LRF and a "potential" generated from the sum of the squares of the differences between the convolved target distribution and the input distribution. Minimising this potential yields a target distribution which when convolved with the LRF matches the raw data. In the ideal case this process would yield a series of delta functions for any given X-ray line pulse height spectrum.

The minimisation of the potential is achieved by rejecting any grain which increases its value. Since the process can easily be trapped in a local minimum (especially in the early stages) positive excursions of the potential are permitted on a random basis controlled by a Boltzman factor which is characterised by a "temperature". As grains are added (and discarded) this temperature is periodically reduced to restrict the positive excursions. This is the "annealing" concept which ensures a smooth decline of the potential and the convergence of the target distribution to a stable solution. Convergence is further aided by reducing the grain size as the process proceeds. For the $\mathrm{x}$-ray spectra under study the number of grains used is typically $10^{6}$ or more. The program is written in compiled BASIC and runs $10^{6}$ grains in about one minute on a Celeron $433 \mathrm{MHz}$ PC.

Figure 10 shows the enhanced output of the SA program in response to the $u$ spectrum of figure 5 using the gaussian LRF derived from the fit in figure 5. The four lines of the $\mathrm{Mn} \mathrm{K}$ radiation in an argon-filled detector are clearly resolved. The number of grains (GN) is $10^{6}$ and the initial temperature (SKT) is $3 \times 10^{-5}$. The high frequency statistical noise present in the peaks is an artefact caused by the "freezing in" of the poor statistics at the beginning of the annealing process. This is different each time it is run and is much reduced by averaging the results of a number of runs (eight replicates seem to work very well). Alternatively, because the frequency of the noise is high, one can simply smooth the data with a gaussian of about half the width of the enhanced peaks. This gives noise-free data with little sacrifice of resolution.

Figure 11 shows the smoothed version of figure 10, transformed back into $x$-space (PHA channels) and compared with the raw data of figure 1. The "energy resolution" of the lines produced by the SA algorithm is $239 \mathrm{eV}$ (FWHM) which compares with $842 \mathrm{eV}$ for the $\mathrm{K}_{\alpha}$ line in the original data. A further improvement is that in the SAenhanced spectrum the width of the peaks is essentially independent of energy. 
Comparing the line positions identified by the peaks in figure 11 with the known line energies shows that they are proportional and agree well with the values obtained from the LN fits (figure 12).

A comparison of the line amplitudes determined from the SA-enhanced spectrum with those obtained from the LN fits is presented in figure 13. The agreement is good for three of the lines but there is a deficit in the $\mathrm{Mn} \mathrm{K}_{\beta}$ line. This is a systematic effect caused by an interference effect between this line and its large neighbour and represents a defect of the present algorithm which it is hoped to correct in due course. The error bars on the data points in figure 13 represent the statistical noise in the LN fits to the line amplitudes.

\section{Conclusions}

It has been demonstrated in this work that the stable line response function produced by a gas microstrip detector operated in "wall-less" mode permits mathematical manipulation which greatly facilitates analysis of the data and explicit enhancement of x-ray line spectra. As a result, effective energy resolution comparable to that of a cryogenic silicon or germanium detector may be achieved. The transformation of the observed pulse height spectrum into the space defined by $u=x^{1 / 2}$ (where $x$ is the PHA channel number) generates a spectrum in which the LRF is gaussian and uniform over a wide range of $\mathrm{x}$-ray energies. This facilitates the application of a spectrum-enhancing algorithm, for which the simulated annealing process is offered as an example.

Spectrum enhancement offers the ability to identify unknown x-ray lines, which can subsequently be quantified accurately by fitting lognormal distributions to the raw data as detailed elsewhere [2]. The SA enhancement algorithm (as implemented in these tests) works well for line sources of X-rays but not for extended distributions. Further exploration of the SA process and other approaches, such as the maximum entropy algorithm, are required to fully exploit the potential for enhancement of GMSD x-ray pulse height spectra.

The very high counting rate capabilities of the GMSD can provide the excess counting statistics required for this type of analysis, particularly in the context of experiments on synchrotron radiation facilities. The ready availability of computing power means that enhanced spectra can be available with little delay and almost in real time. In many x-ray spectroscopy studies this combination may well prove competitive with cryogenic semiconductor detectors. 


\section{References}

1. Oed, Nucl. Instr. \& Meth. A263 (1988) 351-359

2. J.E.Bateman, Precision Measurements of X-ray Line Spectra by Energy Dispersion in a Gas Microstrip Detector, to be published

3. J.E. Bateman, J.F.Connolly, G.E.Derbyshire, D.M.Duxbury, J.Lipp, J.A.Mir, R. Stephenson, J.E.Simmons and E.J.Spill, Rutherford Laboratory Report, RAL-TR1999-057, (http://www-dienst.rl.ac.uk/library/1999/tr/raltr-1999057.pdf)

4. J.E. Bateman, J.F. Connolly, G.E. Derbyshire, D.M. Duxbury, J. Lipp, J.A. Mir, R. Stephenson, J.E. Simmons and E.J. Spill, R.C.Farrow, B.R.Dobson and A.D.Smith, Gas Microstrip X-ray Detectors for Applications in Synchrotron Radiation Experiments, Rutherford Appleton Laboratory Report, RAL-TR-1999056, (http://www-dienst.rl.ac.uk/library/1999/tr/raltr-1999056.pdf) Presented at the International Workshop on Micro-Pattern Gas Detectors, Orsay, France, 2830 June 1999.

5. EasyPlot for Microsoft Windows V 4.0 by Spiral Software

6. J.E.Bateman, J F Connolly, D.M.Duxbury, G.E.Derbyshire, J.A.Mir, E.J.Spill and R.Stephenson, Energy Resolution in X-ray Detecting Gas Microstrip Detectors, to be published.

7. J E Bateman, J F Connolly, R Stephenson, M Edwards, and J C Thompson, Nucl Instr \& Meth A348 (1994) 372

8. S. Kirkpatrick, C.D. Gelatt and M.P. Vecci, Science 220 (1983) 671-80.

\section{Figure Captions}

1. The pulse height distribution observed in a GMSD operated in "wall-less" mode when it is exposed to the $\mathrm{Mn} \mathrm{x}$-ray lines from an ${ }^{55} \mathrm{Fe}$ source. The gas filling is argon $+25 \%$ isobutane. The fits of lognormal line response functions are also shown.

2. The energy resolution (relative FWHM) measured from a series of $x$-ray lines using a wall-less GMSD and lognormal fits.

3. Simulated $\mathrm{x}$-ray pulse height spectra of $\mathrm{x}$-rays in a GMSD with a typical energy resolution. The x-ray energies are $0.75,1.5,3.0$ and $6.0 \mathrm{keV}$.

4. A U-transform of the pulse height spectrum of figure 3.

5. A U-transform of the pulse height spectrum of figure 1 with gaussian fits to the $\mathrm{x}$ ray lines. 
6. A plot of $\mathrm{c}^{2}$ versus the known line energies where $\mathrm{c}$ is the centroid of each gaussian fit in figure 5 .

7. The U-transform of the pulse height spectrum obtained in a GMSD in reponse to irradiation by a beam of $\mathrm{Ag}$ fluorescent $\mathrm{X}$-rays. The gaussian fits to the various $\mathrm{X}$ ray lines are shown.

8. A plot of $c^{2}$ against the known line energies for the fits of figure 7 .

9. The U-transform of the pulse height spectrum obtained in a GMSD when exposed to fluorescence from a glass slide in air irradiated by an ${ }^{55} \mathrm{Fe}$ source. Si, Ar and $\mathrm{Mn} \mathrm{K}$ lines are the main features.

10. The direct output spectrum (in u-space) of the simulated annealing program when it processes the pulse height spectrum of figure 5 (Mn K lines).

11. The simulated annealing-enchanced pulse height spectrum of figure 10 smoothed and transformed back into $\mathrm{x}$-space for comparison with the original data.

12. The centroids of the LN fits of figure 1 and those of the SA-enhanced spectrum of figure 11 are plotted against the known line energies.

13. The amplitudes (total counts) of the four lines from the ${ }^{55} \mathrm{Fe}$ source obtained from the SA-enhanced data and the LN fits are correlated in this graph. 
FIGURE 1

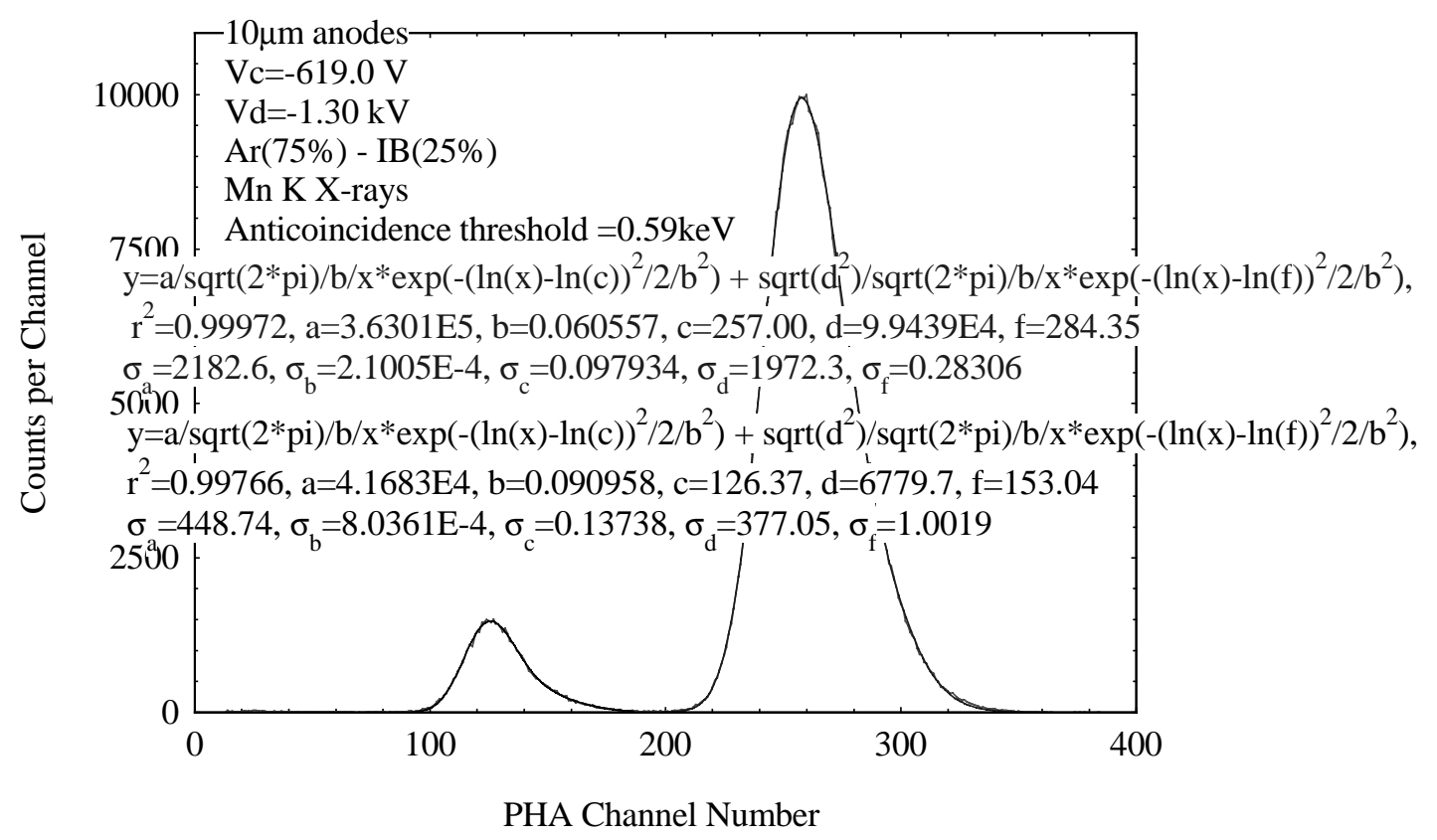

FIGURE 2

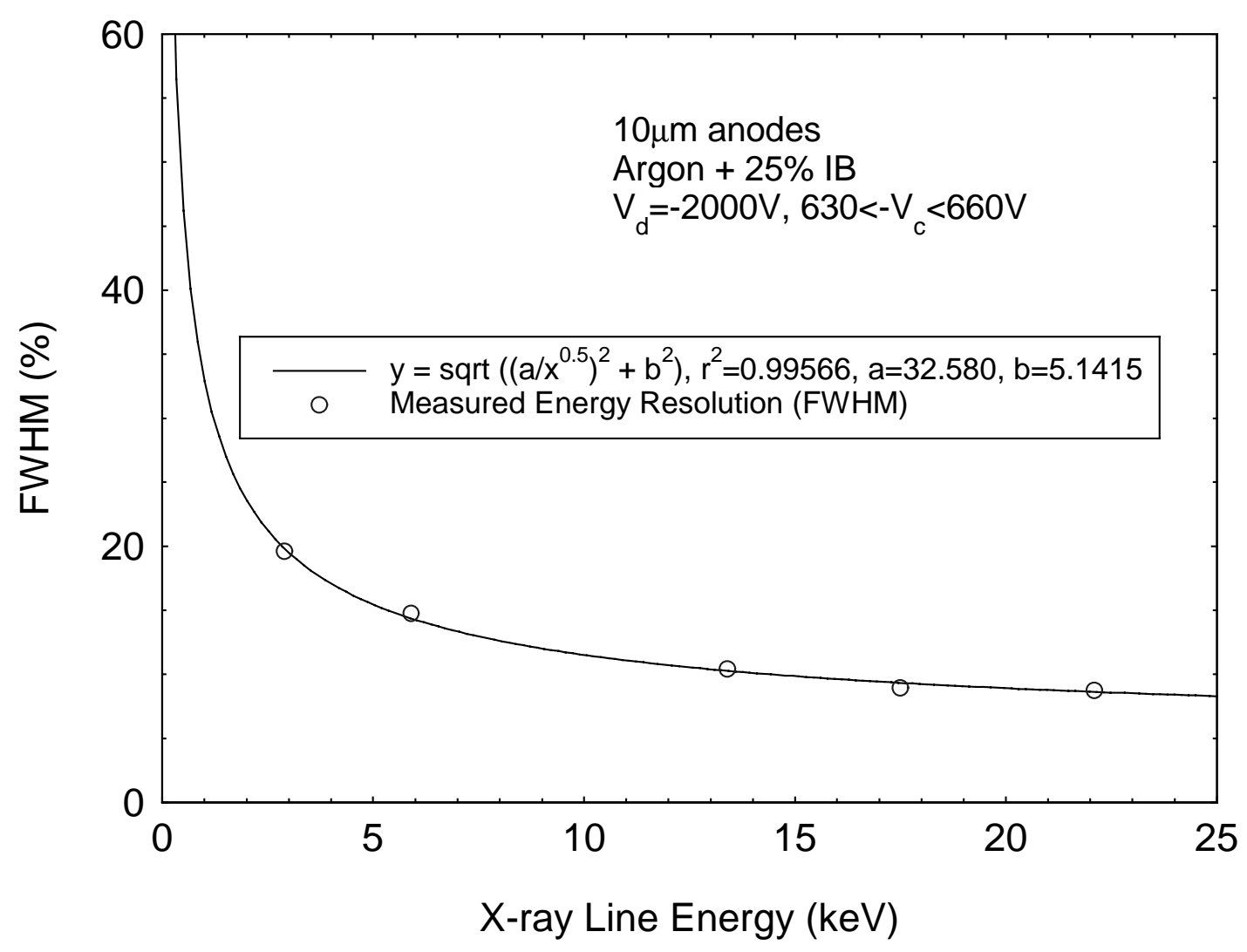


FIGURE 3

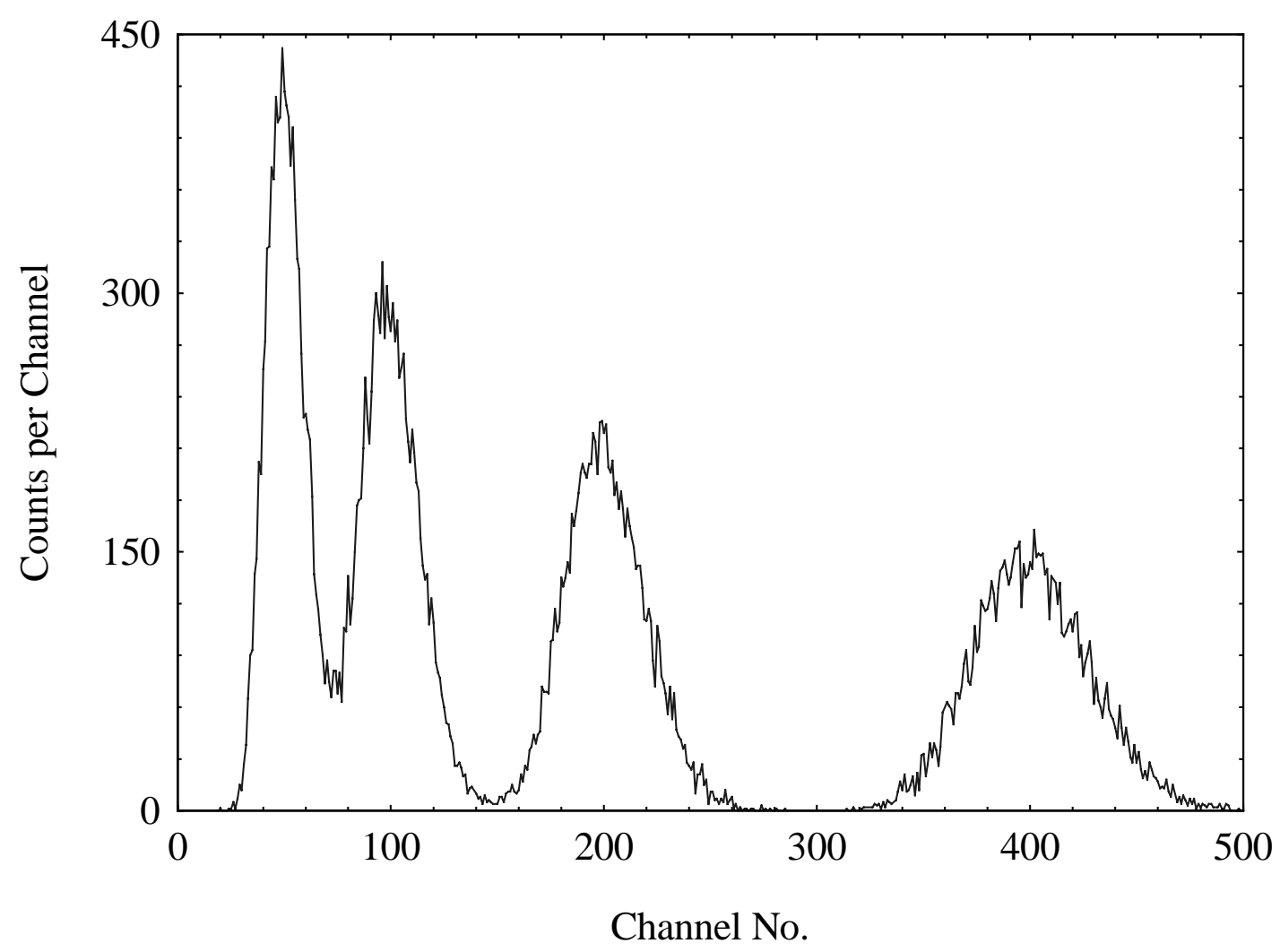

FIGURE 4

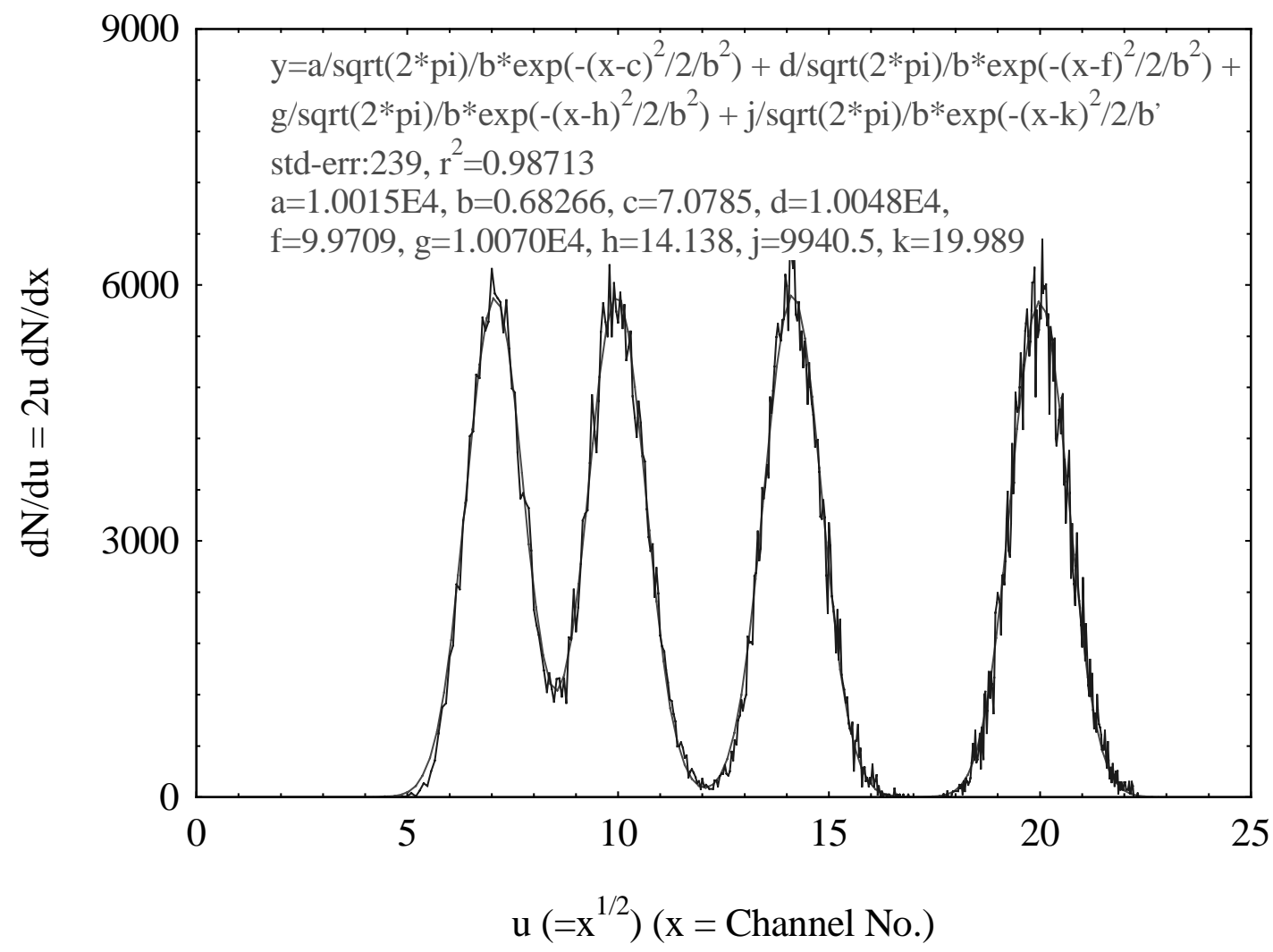


FIGURE 5

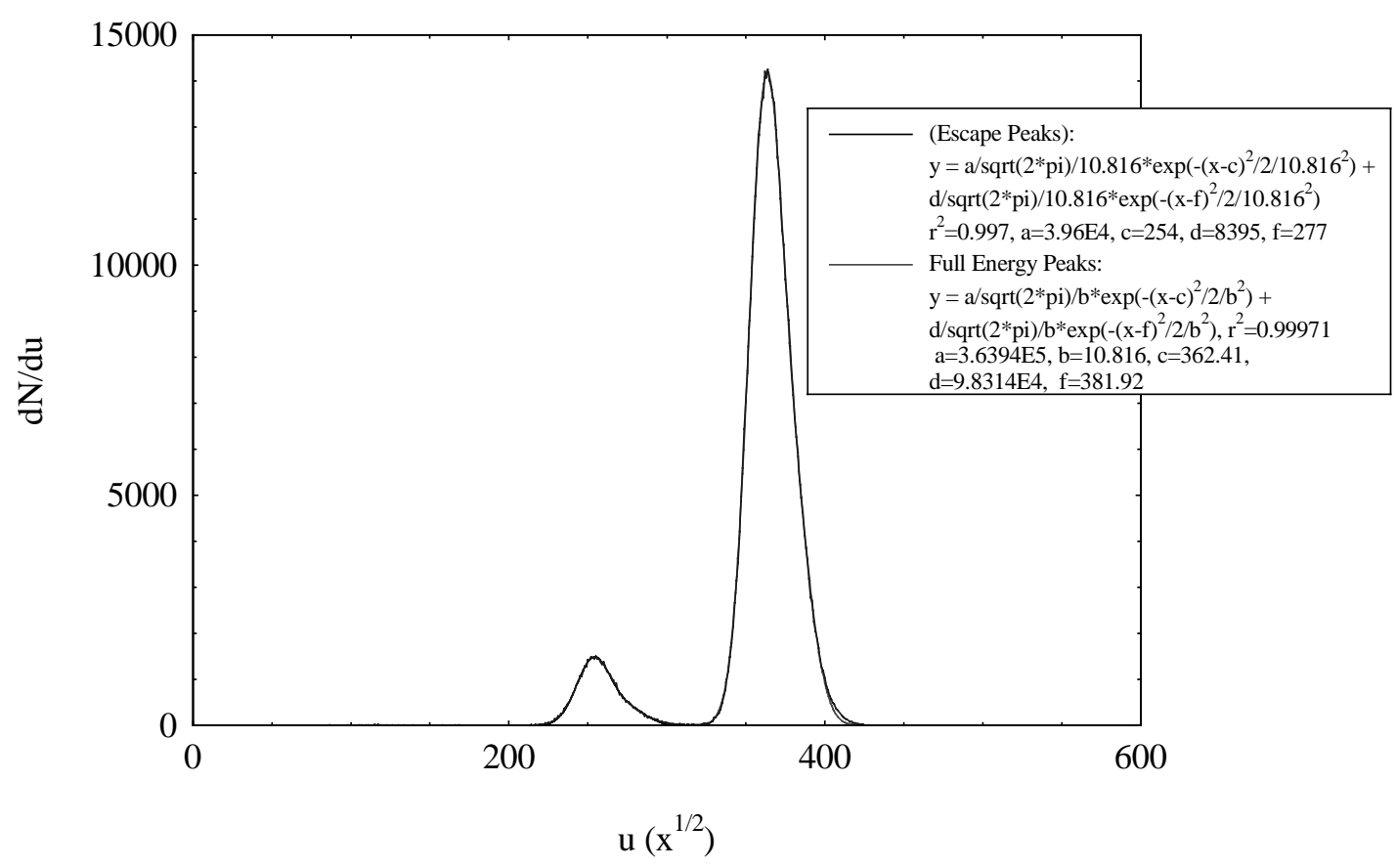

FIGURE 6

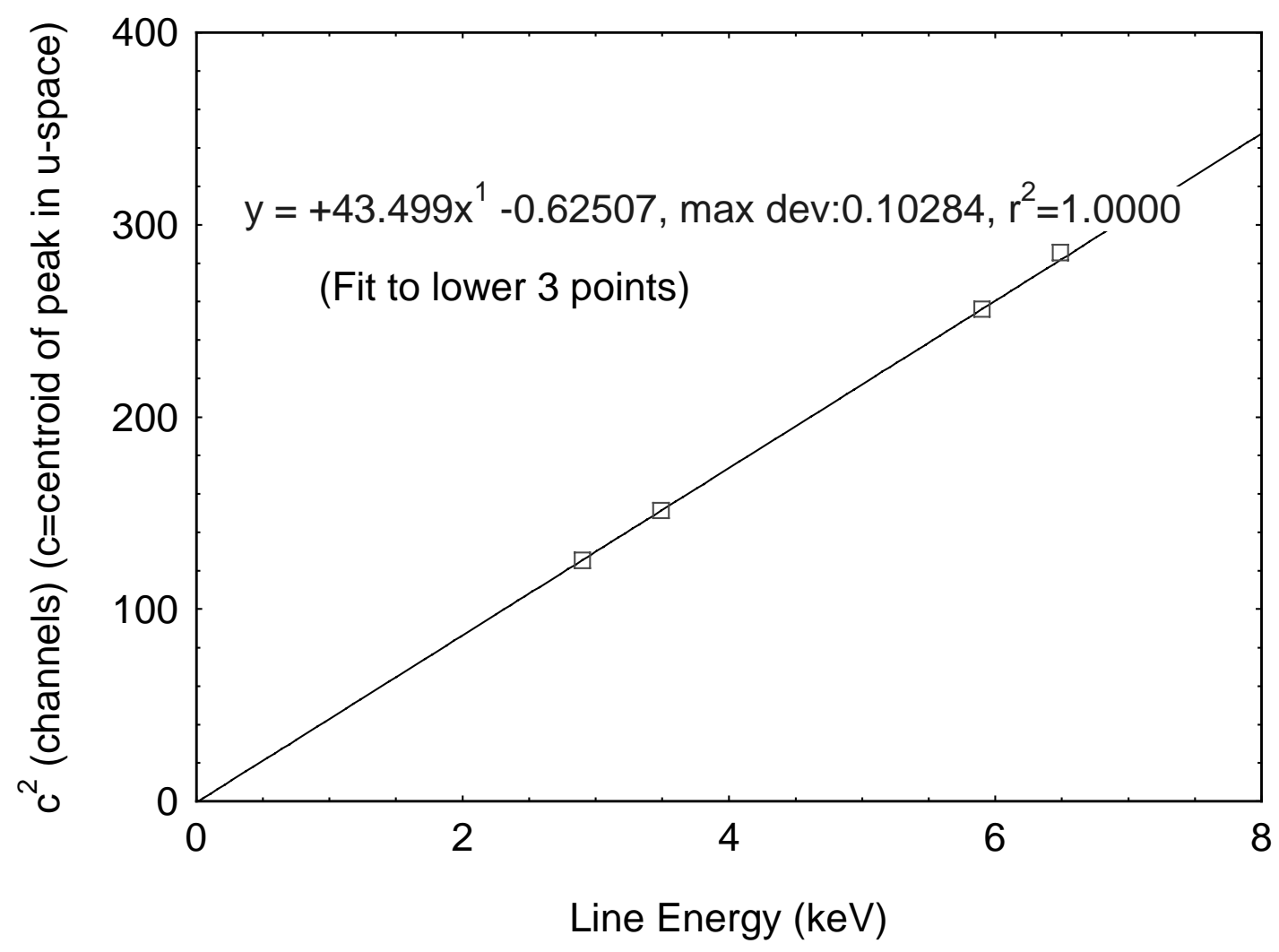


FIGURE 7

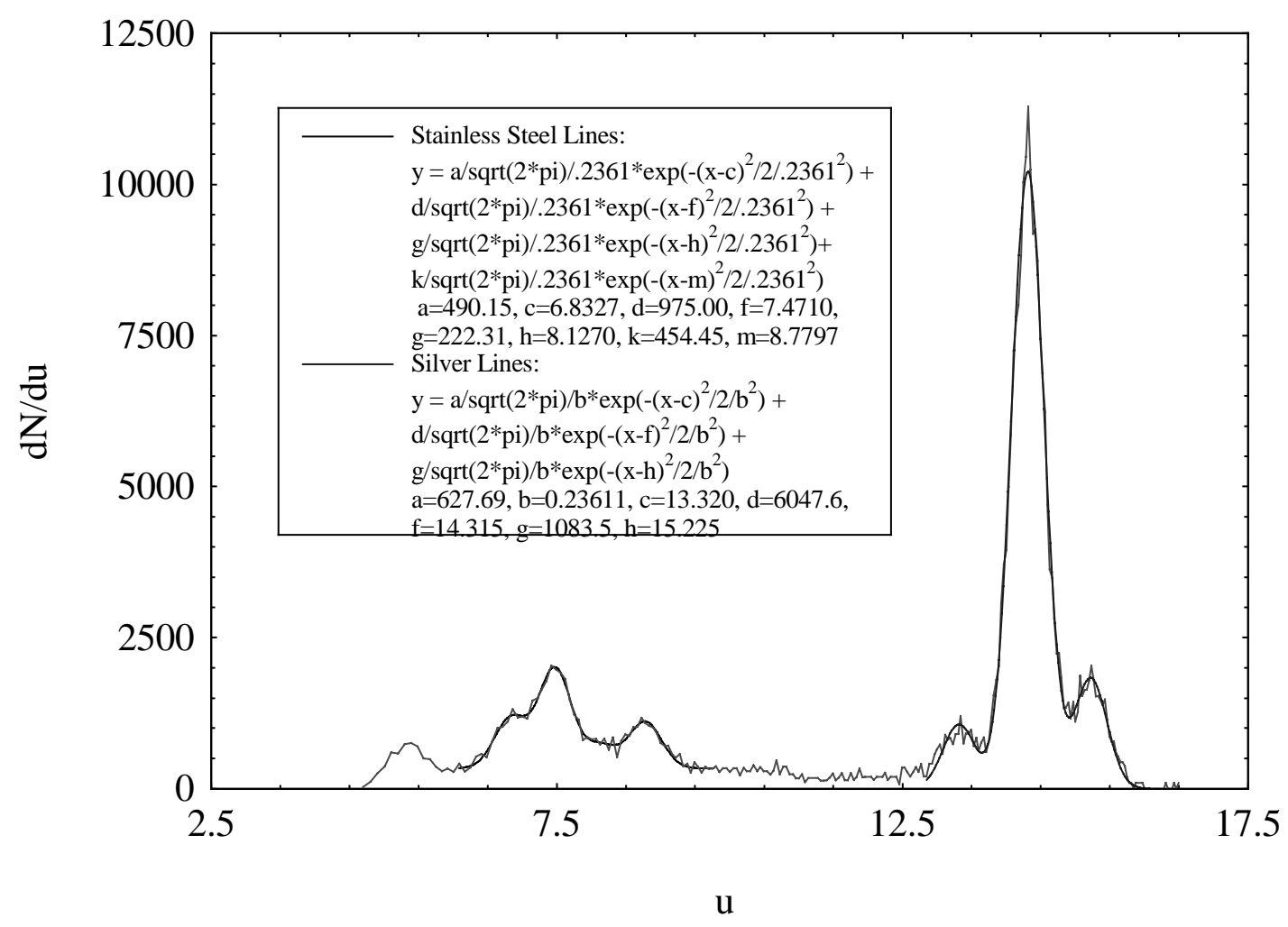

FIGURE 8

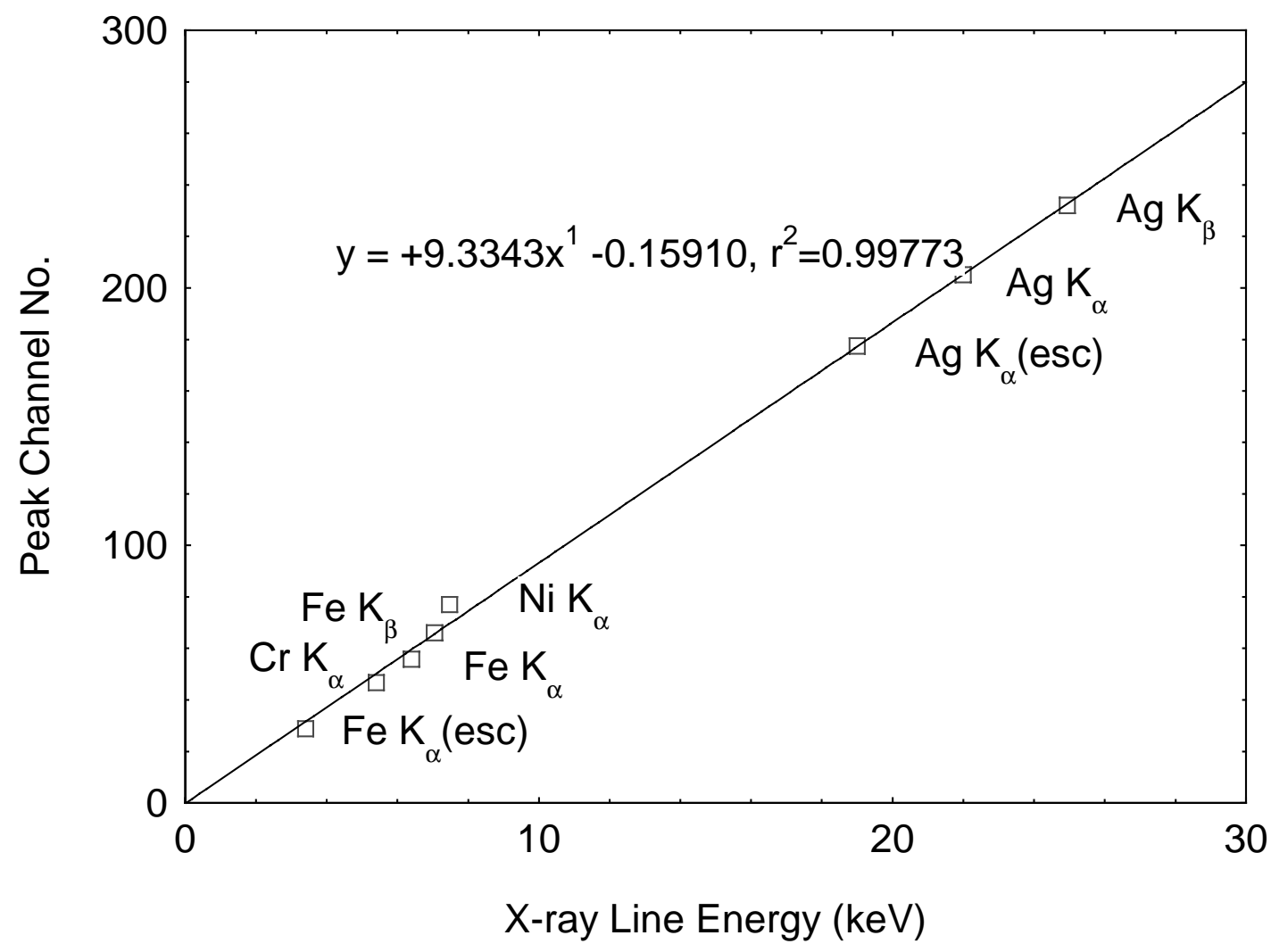


FIGURE 9

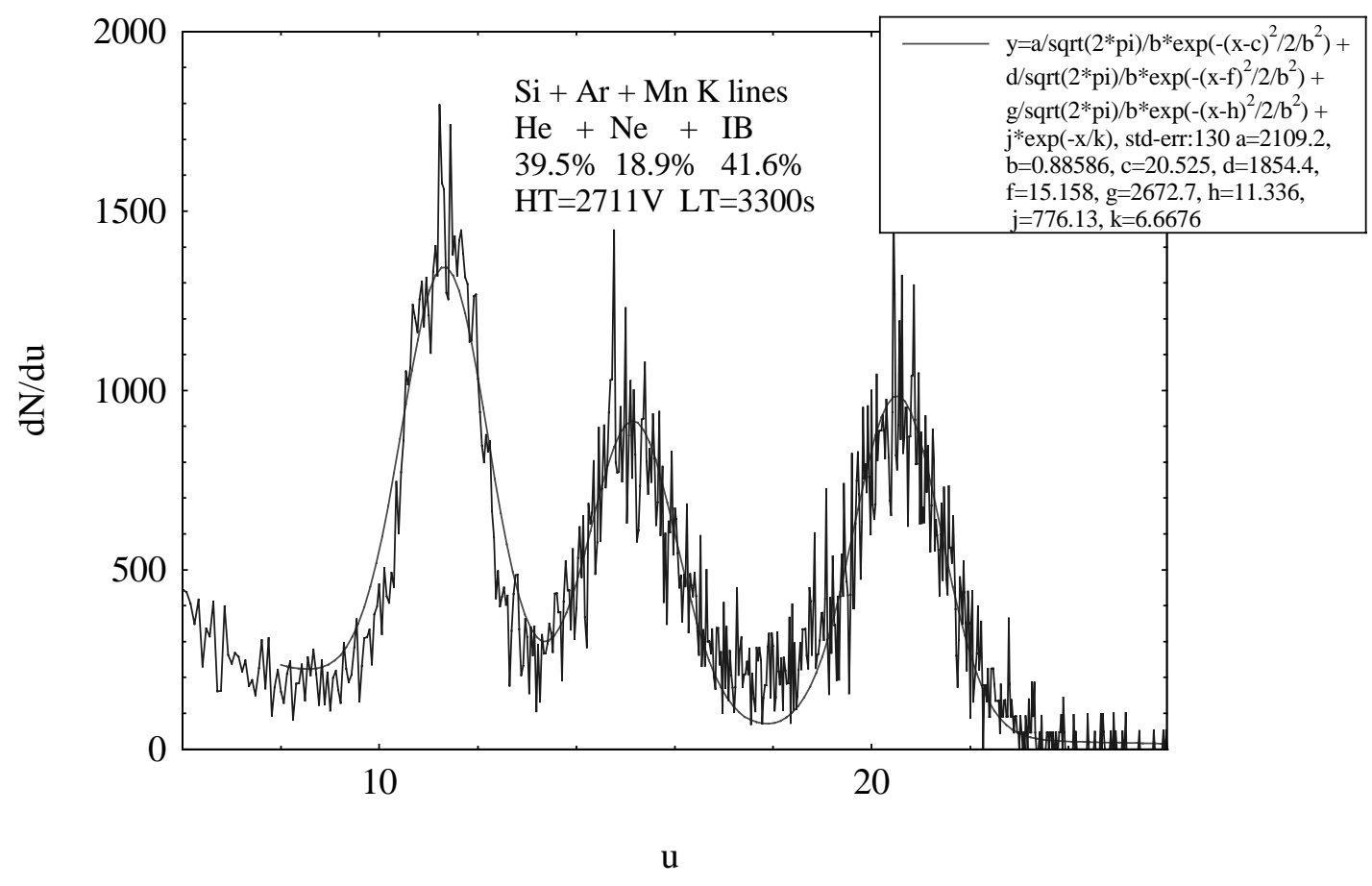

FIGURE 10

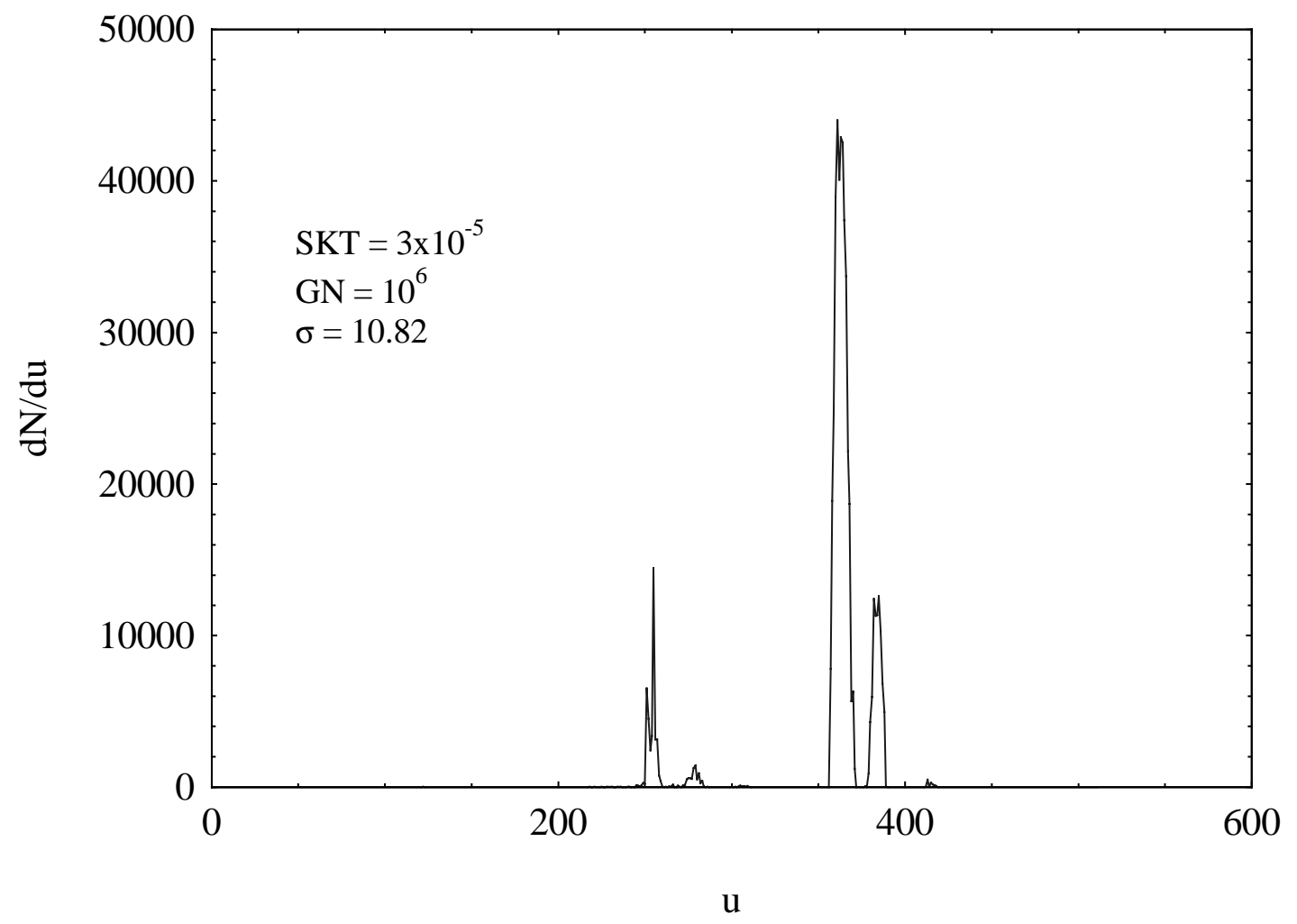


FIGURE 11

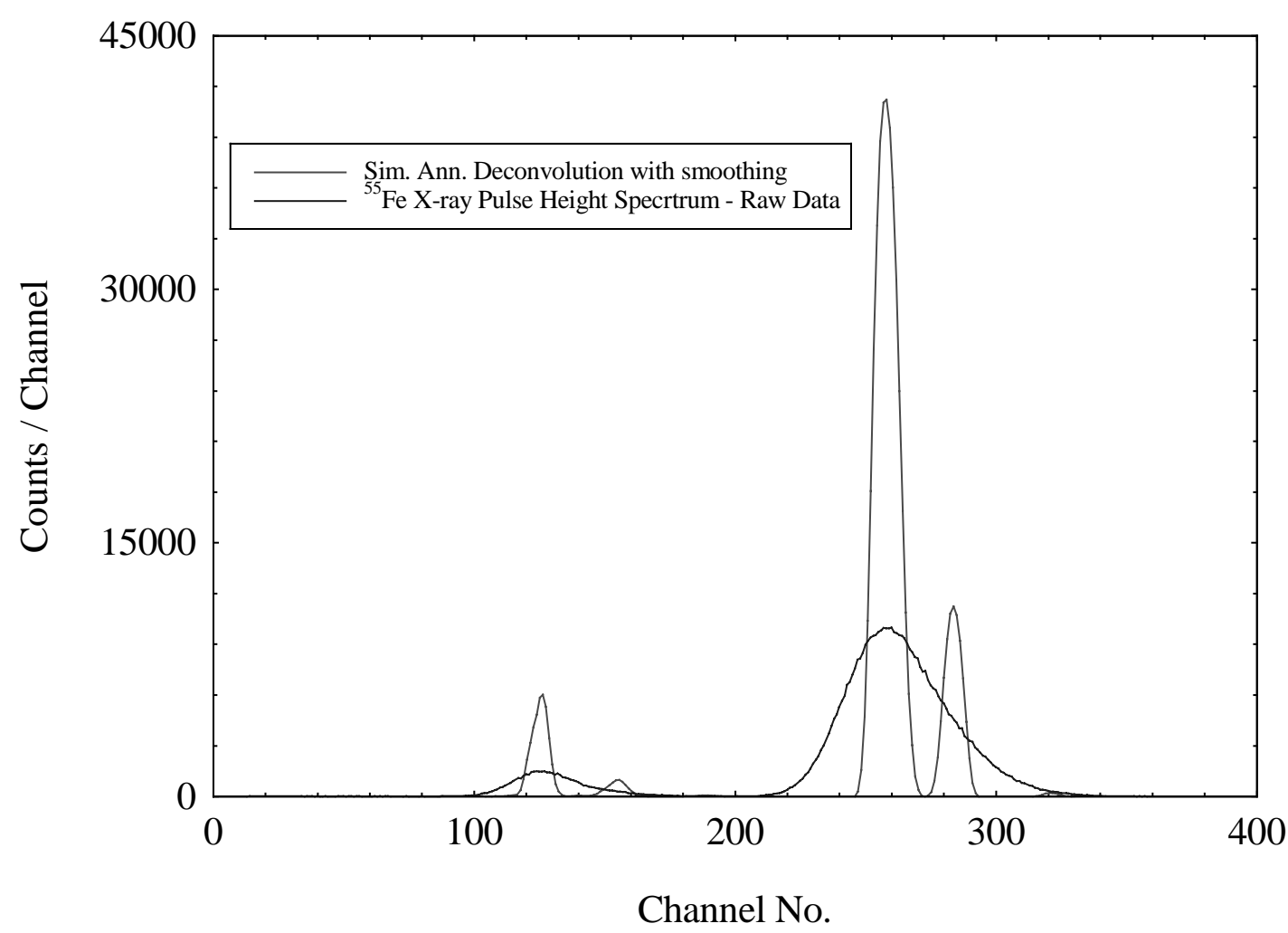

FIGURE 12

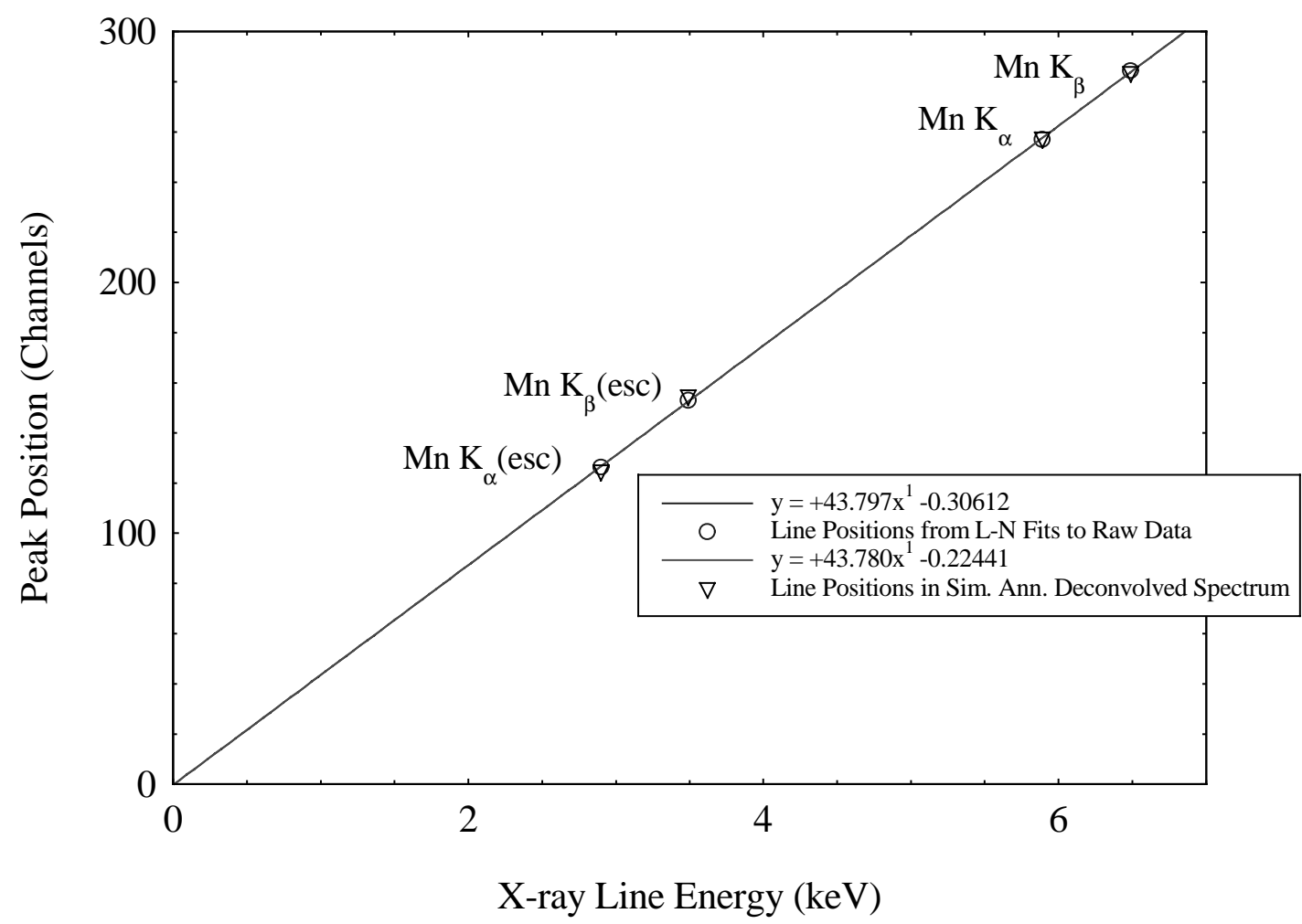


FIGURE 13

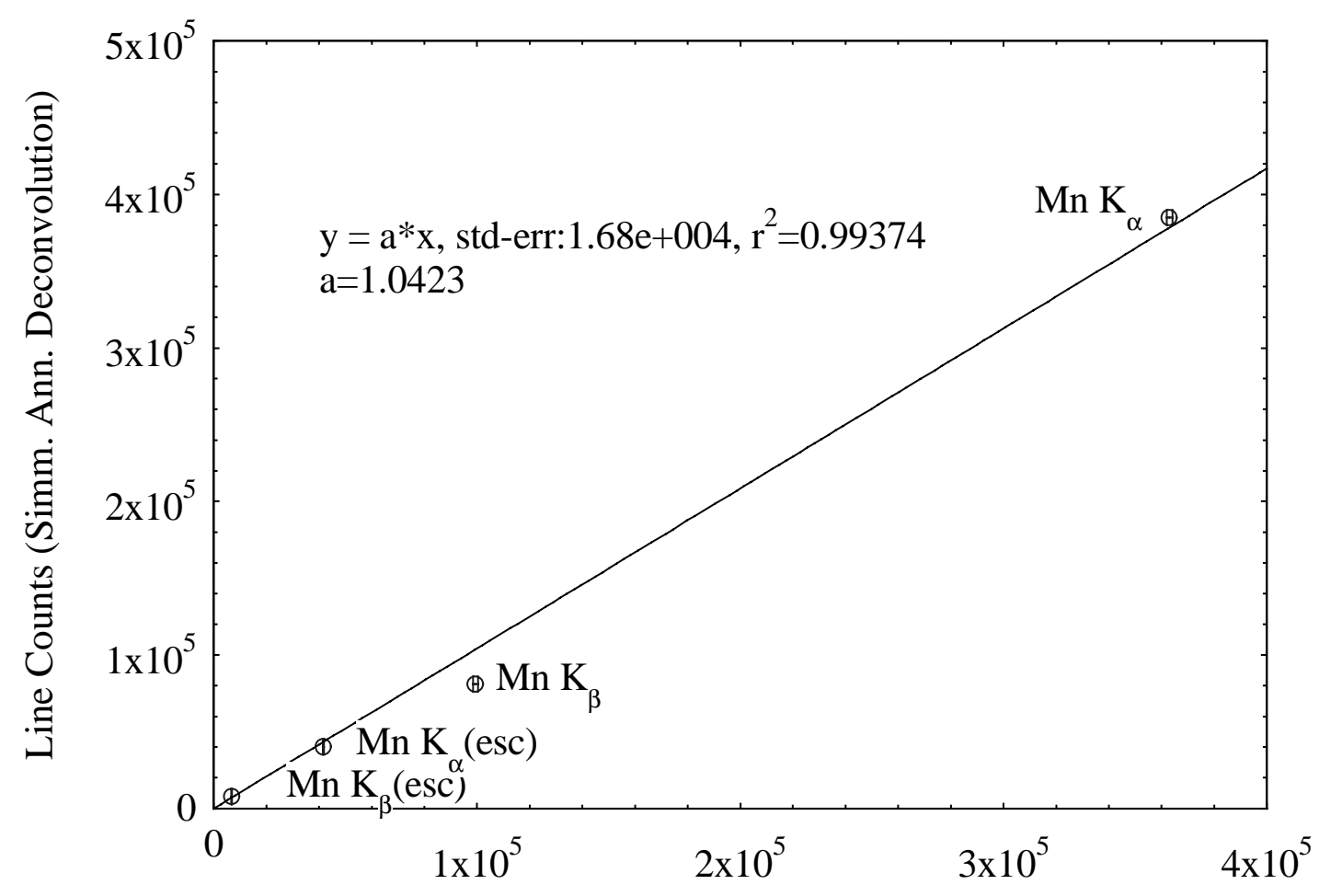

Line Counts (L-N Fits to Raw Data) 